\title{
Chirurgische Versorgung von Bissverletzungen
}

\author{
Claudette Claure Cubillos
}

\section{Indikationen}

Bissverletzungen sind einer der häufigsten Vorstellungsgründe in der tierärztlichen Praxis, die einer chirurgischen Wundversorgung bedürfen. Am häufigsten erfolgen Bisse im Bereich des Kopfes, des Nackens und der Gliedmaßen durch andere Hunde, eher selten durch andere Tiere.

Im Bereich des Brustkorbs und der Bauchhöhle kann es durch Bisse auch zu Schädigungen von inneren Organen kommen. Da Hunde ihre Beute greifen und schütteln, kommt es sehr häufig in der Tiefe zu deutlich schwereren Verletzungen als von außen ersichtlich. Eine Bissverletzung stellt aufgrund der Keimbelastung immer eine infizierte Wunde dar, daher sind eine operative Versorgung und das Einlegen einer Drainage bei größeren Wundhöhlen für einen optimalen Heilungsverlauf unabdingbar.

\section{Erstmaßnahmen}

Nach der allgemeinen Untersuchung und einer ggf. notwendigen Stabilisierung des Patienten sollten die Bisswunden ausgeschoren und gereinigt werden. Das Sondieren der Wunden, um das genaue Ausmaß der Verletzungen festzustellen, kann mit einer Knopfkanüle oder einem Sondierstab erfolgen. Da Bissverletzungen häufig eine größere Wundhöhle haben als an der Oberfläche sichtbar, ist ein großflächiges Ausscheren empfehlenswert. Das Tier sollte immer nach einem Gegenbiss abgesucht werden. Eine Verletzung der Körperhöhlen (Brusthöhle, Bauchhöhle) und der inneren Organe sollte vor der Versorgung der Wunde durch ggf. weitere Diagnostik (Röntgen, Ultraschall) ausgeschlossen werden.

\section{Material}

- steriles OP-Besteck

- Abdecktuch

- Knopfkanüle

- scharfer Löffel

- Spüllösung (sterile $\mathrm{NaCl}$ 0,9\%, ggf. Jodlösung)

- Spülspritze

- Drainage (aktiv/passiv)

- Nahtmaterial (resorbierbar, nicht resorbierbar)

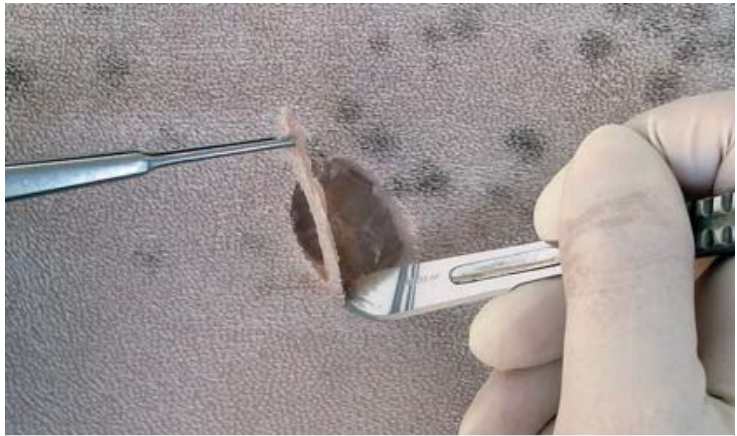

- Abb. 1 Nach Abdecken des Wundgebiets werden die Wundränder aufgefrischt und die Haut so weit wie notwendig eröffnet, um die gesamte Wundhöhle explorieren zu können. @ Klinik für Kleintiere, Universität Leipzig

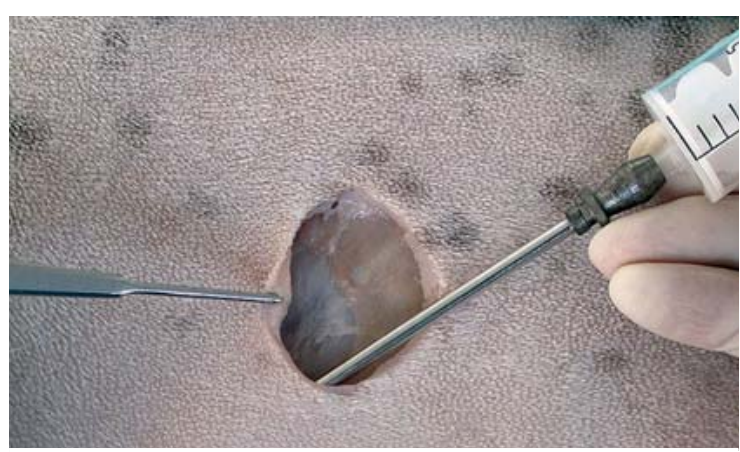

- Abb. 2 Die Wunde wird sondiert, um die Ausmaße darzustellen. @ Klinik für Kleintiere, Universität Leipzig

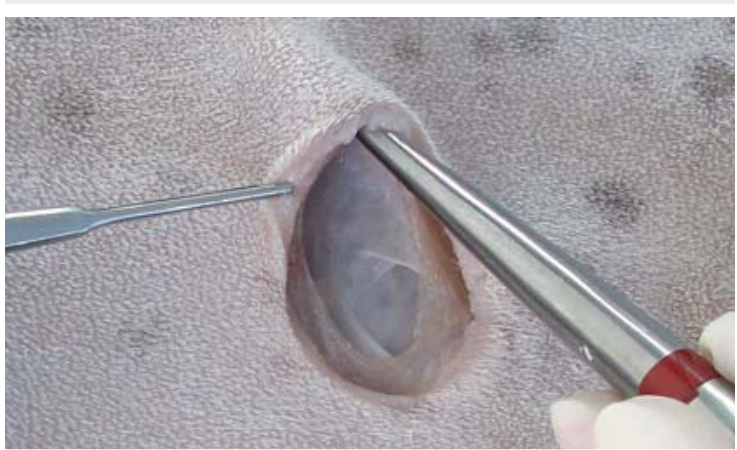

- Abb. 3 Zerstörtes Gewebe, Schmutz und Fremdkörper werden mit dem scharfen Löffel bis zum gesunden Gewebe kürettiert. Um den Druck beim Spülen zu erhöhen, wird eine Knopfkanüle oder Kanüle verwendet. Die optimale Keimreduktion wird hierbei sowohl mechanisch als auch durch den Verdünnungseffekt erreicht. (c) Klinik für Kleintiere, Universität Leipzig 


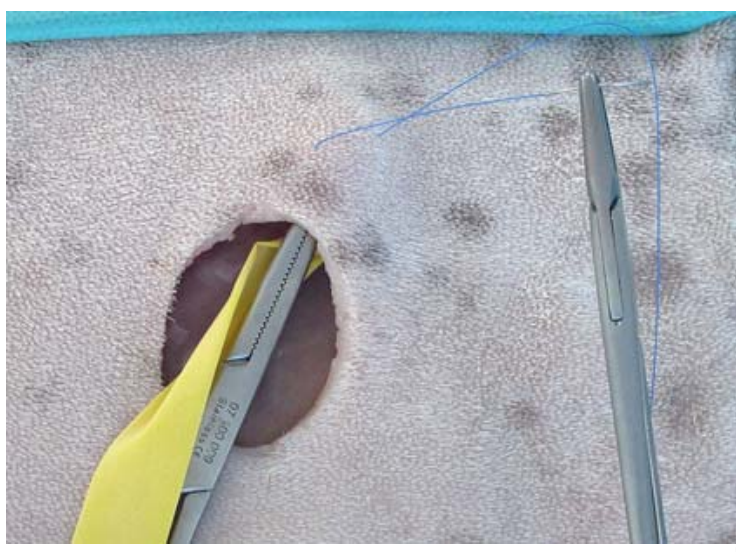

- Abb. 4 Die Drainage wird im dorsalsten Bereich der Wundhöhle mit nicht resorbierbarem Nahtmaterial fixiert. Es ist zu beachten, dass die Fixation der Drainage seitlich der Wundnaht erfolgt. @ Klinik für Kleintiere, Universität Leipzig

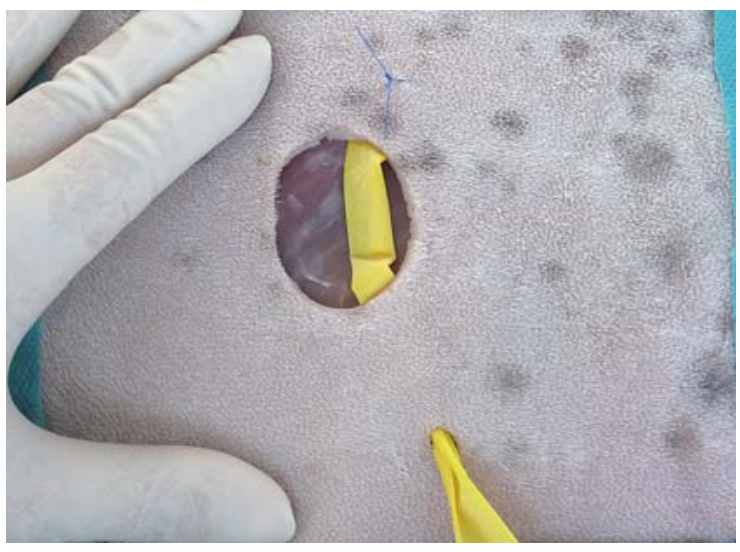

- Abb. 6 Der Ausführungsgang wird hierbei offen belassen. Die Drainage kann abschließend gekürzt werden. ๔c) Klinik für Kleintiere, Universität Leipzig

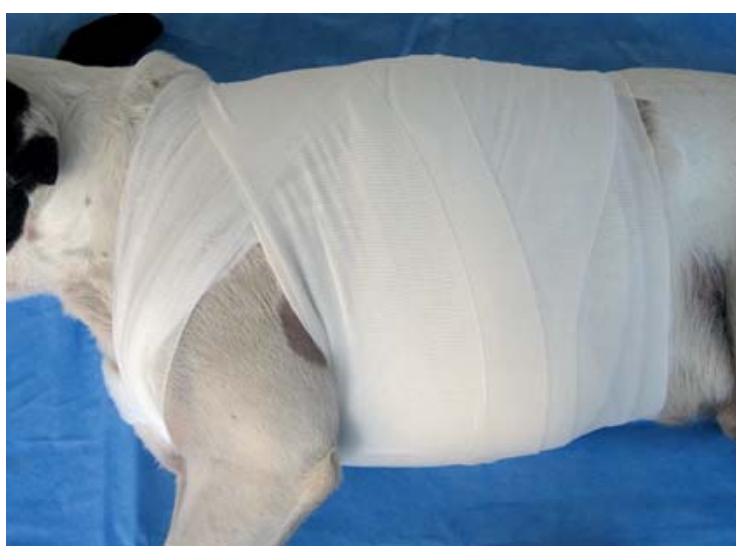

- Abb. 8 Die Wunde und die Drainage sollten mit einem Verband fixiert und vor Manipulation des Patienten geschützt werden. @ Klinik für Kleintiere, Universität Leipzig

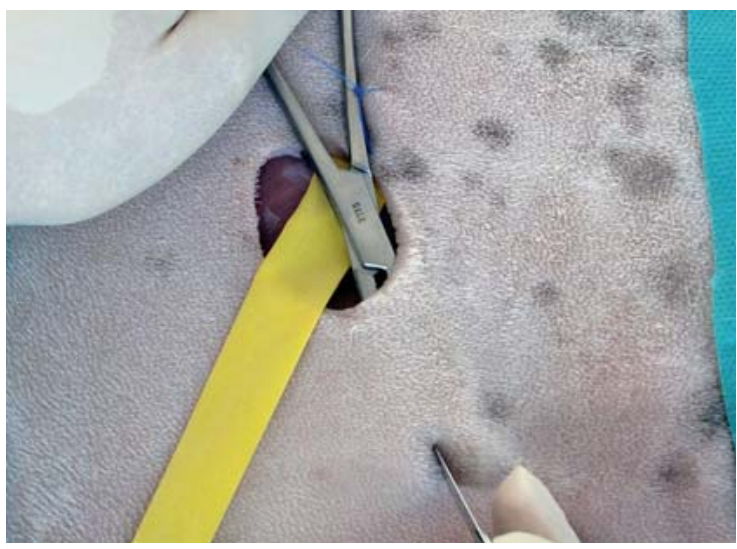

- Abb. 5 Im ventralsten Bereich der Wundhöhle wird ein zusätzlicher Ausführungsgang für die Drainage geschaffen. Diese wird hier erneut mit nicht resorbierbarem Nahtmaterial fixiert. @ Klinik für Kleintiere, Universität Leipzig

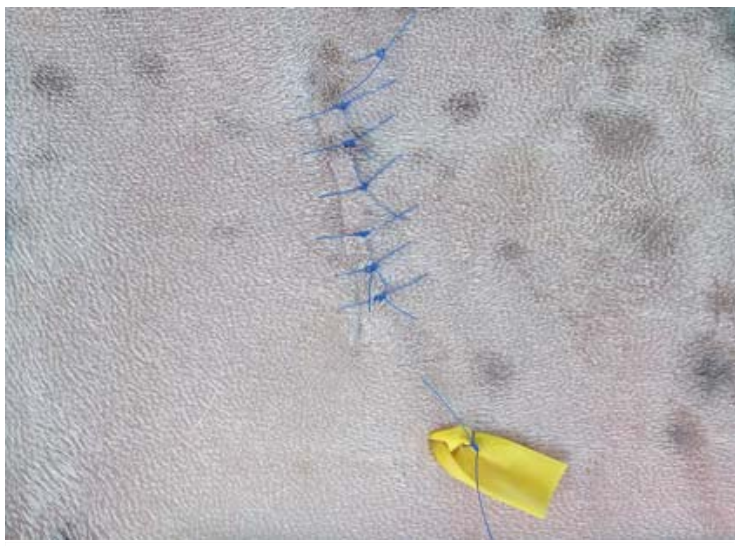

- Abb. 7 Schichtweiser Wundverschluss der Unterhaut und der Haut unter besonderer Berücksichtigung der Drainage. Die Knoten der Hauthefte sollten nicht auf der Wundnaht liegen. @ Klinik für Kleintiere, Universität Leipzig

Korrespondenzadresse

\section{Tierärztin Claudette Claure Cubillos}

Abteilung Chirurgie, Klinik für Kleintiere

Universität Leipzig

An den Tierkliniken 23

04103 Leipzig

Claudette.Cubillos@kleintierklinik.uni-leipzig.de

\section{Bibliografie}

DOI https://doi.org/10.1055/a-0599-7147

kleintier konkret 2018; 21: 42-43

(c) Georg Thieme Verlag KG Stuttgart · New York

ISSN 1434-9132 